\title{
Apoptotic Effects of Temozolomide and Naturopathic Agents upon Glioblastoma Cells
}

\author{
Albert Magro ${ }^{1 *}$, Alice Magro ${ }^{1}$, Sirish Shrestha ${ }^{1}$ and Kathy Brundage ${ }^{2}$ \\ ${ }^{1}$ Fairmont State University, USA \\ ${ }^{2}$ Microbiology, Immunology and Cell Biology, West Virginia University, USA
}

\begin{abstract}
Temozolomide (TMZ), thymoquinone (TMQ), epigallocatechin gallate (EPIGAL) and staurosporine (STAURO) were used as apoptotic inducing agents acting upon the U87-MG (ATCC, HTB15), LN18 (ATCC, CRL2610) and U118-MG (ATCC, HTB14) glioblastoma multiforme (GBM) cell lines. TMZ is the current drug of choice for primary treatment and adjuvant therapy for recurrent GBM. TMQ and EPIGAL are naturopathic agents, while STAURO is a well-studied apoptotic-inducing agent. The degree and time course of apoptosis were measured by flow cytometry techniques capable of detecting changes in mitochondrial function using the fluorescent dye MitoTracker Deep Red. Phosphatidylserine exposure and plasma membrane permeability were detected simultaneously using violet fluorescent reactive dye (VFRD) in combination with AnnexinV-488. The apoptotic effectiveness of the inducing agents TMZ, TMQ, EPIGAL and STAURO were compared with their ability to inhibit invasiveness and degrade Class I major histocompatibility complex (MHC) determinants. Invasiveness was measured in vitro by the 3D matrigel spheroid invasion assay. The density of the class $1 \mathrm{MHC}$ determinants was measured by flow cytometry. Although TMZ is widely used for the chemotherapeutic treatment of GBM, it was determined that the time course for TMZ induced apoptosis was slower than those of TMQ, EPIGAL and STAURO. Unexpectedly, TMZ was ineffective in its ability to inhibit in vitro invasiveness and did not degrade the class I MHC determinants as effectively as the other apoptotic inducing agents. The findings raise the question of whether in vitro assays of apoptosis and invasiveness are the best measures of the effectiveness of chemotherapeutic agents for the primary treatment and adjuvant therapy of recurrent GBM. The findings also point to the in vivo complexity of the efficacy of chemotherapeutic agents whereby preserving the components of natural and acquired immune mechanisms may be more important than the rapid apoptotic effects of the chemotherapeutic agent.
\end{abstract}

Keywords: Chemotherapeutic agents; Epigallocatechin gallate; Glioblastoma multiforme; Immunosuppression; Invasiveness; Major histocompatibility complex; Naturopathic; Temozolomide; Thymoquinone

\section{Introduction}

Therapeutic regimens for glioblastoma multiforme (GBM) and other high-grade gliomas have fallen short of providing effective treatment. Clinical studies comparing chemotherapeutic agents have indicated increased tumor shrinkage and a very slight increase in median survival times, but no evidence for an increase in survival rates [1-4]. More recent clinical studies employing surgery with the use of chemotherapeutic regimens in combination with radiation therapy or by receptor mediated growth hormone deprivation are slightly more effective in increasing median survival times, but again have had limited success in increasing the overall survival rate in patients being treated for primary or recurrent glioblastomas [5-6]. New treatments are diligently being pursued and provide hope for effective treatment $[7,8]$. However, the difficulties of complete resection, the resistance to radiation and other therapies and particularly the intractable malignant invasiveness still remains at the root of the very poor survival prognosis for patients with GBM and other high grade gliomas. The fact that GBMs are usually malignantly invasive, but in general do not metastasize, is what renders studies about invasiveness particularly relevant to glioblastomas [9]. It is well known that all immunological, radiological and pharmacological treatments of GBMs are treatments that induce cell death by apoptosis. In fact, other than surgery, malignant cell types require a clinical treatment that induces cell death by apoptosis because the cellular debris and inflammation produced by massive necrosis or necroptosis of an extensive tumor would be systemically injurious to the patient. This raises the question of whether effective apoptotic inducing agents also have the property of inhibiting the invasiveness of GBMs. An additional question, is there a correlation between the ability of a chemotherapeutic agent to induce apoptosis and to inhibit the invasiveness of GMB?

The DNA alkylating agent temozolomide (TMZ) is a secondgeneration imidazotetrazin that when administered p.o. enters the cerebrospinal fluid and does not require hepatic metabolism for activation. It is the current drug of choice for primary treatment and adjuvant therapy for recurrent glioblastoma multiforme [10-17]. A point of interest is to test the ability of temozolomide to induce apoptosis and inhibit the invasiveness of GMBs as compared to other agents capable of inducing apoptosis.

Staurosporine (STAURO) has been well studied as an apoptoticinducing agent [18] and is relevant to current explorations regarding the efficacy of small-molecule kinase inhibitors in the treatment of solid cancers including glioblastoma [19]. Other compounds of interest are naturopathic agents. Naturopathic approaches to health care use natural, non-toxic herbs and other agents as supplements to support and encourage healing processes. A fertile source of well-tolerated natural antineoplastic agents has been extracts from botanical preparations, including extracted oils, herbal extracts and teas. Such extracts include the natural products thymoquinone [20-23] and epigallocatechin

*Corresponding author: Albert Magro, Fairmont State University, USA, Tel: 3044491328; E-mail: a3magro@aol.com

Received May 26, 2018; Accepted June 11, 2018; Published June 18, 2018

Citation: Magro A, Magro A, Shrestha S, Brundage K (2018) Apoptotic Effects of Temozolomide and Naturopathic Agents upon Glioblastoma Cells. J Oncol Transl Res 4: 132. doi: 10.4172/2476-2261.1000132

Copyright: (c) 2018 Magro A, et al. This is an open-access article distributed under the terms of the Creative Commons Attribution License, which permits unrestricted use, distribution, and reproduction in any medium, provided the original author and source are credited. 
gallate [24-28]. Thymoquinone (TMQ) is the bioactive compound derived from black seed oil (Nigella sativa), while epigallocatechin gallate (EPIGAL) is the major polyphenol component of green tea. Both TMQ and EPIGAL are promising antineoplastic agents that are well tolerated at high doses and show low toxicity to non-cancerous cells. STAURO has been well studied and, although not currently a clinically employed drug, it is relevant to current explorations regarding the efficacy of small-molecule kinase inhibitors in the treatment of solid cancers including glioblastoma [29,30]. Although TMQ and EPIGAL are reported to be apoptotic inducing agents [31-36] that cross the blood brain barrier [37-42], their apoptotic effects upon GBMs has not been studied. Also, there are no studies comparing the apoptotic effectiveness of TMZ to the naturopathic agents TMQ and EPIGAL.

As aforementioned, TMZ is the drug of choice in the treatment of GBMs. It would seem that TMZ would be more effective in its ability to induce apoptosis as compared to other agents in the treatment of GBMs. A point of interest is, how does TMZ compare to TMQ EPIGAL and STAURO in their ability to induce apoptosis in established glioblastoma cell lines using a variety of apoptotic indicators?

It is well established that the malignancy of GBMs is in large part due to the propensity of the tumor to invade surrounding brain tissue [43]. Accordingly, of interest is the efficacy of TMZ as compared to TMQ, EPIGAL and STAURO in their ability to inhibit in vitro growth and invasiveness of GBMs.

Deficiencies associated with the Class I major histocompatibility complex (MHC) molecules restrict antigen presentation machinery and thus helps tumor cells evade the classical cell-dependent immune responses [44]. It has been reported, that as GBMs proceed through apoptosis, metalloproteinases and serine proteases are activated which degrade cell surface determinants $[45,46]$. Upon treatment with chemotherapeutic agents, those cells within the tumor that survive the apoptotic process could have a low density of class I MHC molecules on the surface and escape immune surveillance. Thus, an additional question is, how does TMZ compare to TMQ, EPIGAL and STAURO in their ability to degrade HLA-ABC determinants as the glioblastoma cells proceed through apoptosis?

\section{Methods \\ Cell types and maintenance}

All cell lines were obtained from American Type Culture Collection (ATCC). The LN18 cell line (ATCC, CRL-2610) was established in 1976 from a patient with a right temporal lobe glioma [47]. The cells are poorly differentiated, adherent and grow well in culture. The U87MG (ATCC, HTB15) and U118-MG (ATCC, HTB14) glioblastoma cell lines were first isolated from patients afflicted with brain glioblastoma multiformes by Ponten et al. [48]. All three cell lines are considered Grade IV astrocytomas and are transplantable as xenographs into nude mice $[47,49,50]$. The cells are anchorage independent and grow well as monolayers. Cells were maintained at $37^{\circ} \mathrm{C}$ in an atmosphere of $5 \% \mathrm{CO}_{2}$ at $100 \%$ humidity in $75 \mathrm{~cm}^{2}$ flasks in Advanced DMEM (F12) supplemented with the dipeptide L-alanyl-L-glutamine $(2 \mathrm{mM})$, nonessential amino acids, penicillin (100 units/ml), streptomycin $(100 \mu \mathrm{g} /$ $\mathrm{ml})$, amphotericin B $(0.25 \mu \mathrm{g} / \mathrm{ml})$, HEPES $(10 \mathrm{mM})$ and fetal bovine serum (5\%). Cells were sub-cultured by trypsinization (0.25\% trypsin, EDTA). Media and supplements were obtained from Gibco.

\section{Apoptotic inducing agents}

The apoptotic inducing agents TMZ, STAURO, TMQ and EPIGAL were prepared to the appropriate concentrations from a DMSO solubilized stock solution where the reaction concentrations of DMSO were less than $0.03 \%$. Apoptosis was induced at $37^{\circ} \mathrm{C}$ under growth conditions by the addition of TMZ, TMQ and EPIGAL at a reaction concentration of $100 \mu \mathrm{M}$, while STAURO was used at a reaction concentration of $1 \mu \mathrm{M}$ in cells adhered as a monolayer in 24 well flat bottom plates (Costar). The cells were then harvested by the use of enzyme free cell dissociation buffer (Gibco). All apoptotic inducing agents were obtained from Sigma-Aldrich.

\section{Detection of apoptosis}

For the mitochondrial function experiments the 24 well plates consisting of a monolayer of glioblastoma cells were allowed to incubate for $8 \mathrm{~h}$ after the addition of the inducing agents. Apoptosis was detected by changes in the mitochondrial function that was illustrated by changes in fluorescent intensity of the mitochondrial membrane binding dye MitoTracker Deep Red 633 (Thermo Fisher). The $1 \mathrm{mM}$ stock solution of MitoTracker was diluted and added to the apoptotic and non-apoptotic control cells at a cell concentration of $1 \times 10^{6}$ cells/ $\mathrm{ml}$ with a MitoTracker reaction concentration of $300 \mathrm{nM}$. After lifting the cells from the monolayer with enzyme free cell dissociation buffer, the cells were allowed to stain live in suspension at $37^{\circ} \mathrm{C}$ in Advanced DMEM (F12) growth medium under growth conditions, for $20 \mathrm{~min}$. Cells were then harvested and fixed in $1 \%$ paraformaldehyde (Sigma) and subsequently analyzed by flow cytometry.

For apoptosis detected by the Violet Fluorescent Reactive Dye (VFRD) vs. AnnexinV dot plots, 24 well plates consisting of a monolayer of glioblastoma cells were allowed to incubate for $12 \mathrm{~h}$ after the addition of the inducing agents. Apoptosis was detected by AnnexinV binding to membrane-exposed phosphatidylserine in combination with VFRD dye (Thermo Fisher). Following washing and centrifugation the apoptotic and non-apoptotic cells were suspended in $1 \mathrm{ml}$ of PBS each at a cell concentration of $1 \times 10^{6} \mathrm{cells} / \mathrm{ml}$. $1 \mu \mathrm{L}$ of reconstituted VFRD dye was added to the $1 \mathrm{ml}$ of cell suspension, which was then incubated on ice for $30 \mathrm{~min}$ in the dark. The cells were then washed $2 \mathrm{x}$ with $1 \mathrm{ml}$ of PBS and suspended in $1 \mathrm{ml}$ of $10 \mathrm{mM}$ HEPES, $140 \mathrm{mM} \mathrm{NaCl}$ and $2.5 \mathrm{mM}$ $\mathrm{CaCl}_{2}, \mathrm{pH} 7.4$ (annexin-binding buffer). Just prior to FACS analysis $1 \mu \mathrm{L}$ of AnnexinV, Alexa Fluor 488 conjugate (Thermo Fisher) was added to the $1 \mathrm{ml}$ violet stained cell suspension. The doubly stained cells were then assayed by flow cytometry.

\section{Flow cytometry}

The samples were analyzed on the 4 laser LSRFortessa (BD Biosciences) using FACS Diva 8.0 software for data collection. A minimum of 10,000 events were collected per sample. Data analysis was done using FCS Express 6 Flow software (De Novo Software, Los Angelis CA).

\section{D Matrigel Spheroid Invasion Assay}

The effects upon growth and invasiveness were tested using a 3D spheroid assay as measured by the changes in the radius and volume surrounding the spheroid. Spheroid assays were performed in Corning Costar Ultra-Low attachment 96 well round bottom plates. The plates feature a covalently bound hydrogel layer that effectively inhibits cellular attachment. Prior to the introduction of the low growth factor matrigel, 6,000 glioblastoma cells were introduced into the wells of the plate in 50 $\mu \mathrm{L}$ of medium (0.5\% FBS) and incubated overnight to allow spheroid formation. $50 \mu \mathrm{L}$ of low growth factor matrigel that contained $0.4 \mathrm{mg} /$ ml collagen-1 was neutralized with $\mathrm{NaHCO}_{3}$ and then introduced into each well. The matrigel and collagen mix was added ice cold to the ice chilled wells containing the glioblastoma spheroids. The plate was then 
centrifuged at $200 \mathrm{~g}$ for $5 \mathrm{~min}$ at $4^{\circ} \mathrm{C}$. The cells were then incubated at $37^{\circ} \mathrm{C}$ to allow polymerization of the matrigel/collagen-1 mixture. Following polymerization, $100 \mu \mathrm{L}$ of medium (10\% FBS) was gently added to the matrigel surface of each well, with or without apoptotic inducing agents. The apoptotic inducing agents were added in the $100 \mu \mathrm{L}$ addition at twice the concentration of the appropriate reaction concentration in the $200 \mu \mathrm{L}$ well volume. The plate was then incubated for 5 days at $37^{\circ} \mathrm{C}$. Micrographs were taken at $40 \mathrm{x}$ with a Nikon Diaphot inverted microscope equipped with Prog Res Capture imaging software and camera. The areas and average diameters of the spheroid images were calculated using Image J software, which was downloaded from NIH.GOV.

\section{Flow cytometry analysis of HLA-ABC determinants on the surface of glioblastoma cells}

Apoptosis was induced in cells adhered as a monolayer in 24 well plates. The plates were allowed to incubate for $15 \mathrm{~h}$ after the addition of the inducing agents. The cells were then harvested by the use of enzyme free cell dissociation buffer (Gibco). Rabbit purified antibodies for HLA-ABC (Abcam) were used as a primary antibody at a reaction concentration of $5 \mu \mathrm{g} / \mathrm{ml}$. Rabbit purified anti-Keyhole Limpet Hemocyanin (anti KLH, Sigma-Aldrich) was used as the primary antibody negative control $(5 \mu \mathrm{g} / \mathrm{ml})$. The secondary antibody was $\mathrm{F}(\mathrm{ab})_{2}$-goat anti-rabbit IgG conjugated to phycoerythrin at a reaction concentration of $2.5 \mu \mathrm{g} / \mathrm{ml}$ (Jackson ImmunoResearch). Flow cytometry data were then accumulated as described above.

\section{Results}

\section{Changes in mitochondrial function as an indicator of apoptosis}

The histograms of Figure 1 represent mean fluorescence intensity (MFI) of MitoTracker Deep Red 633 dye versus cell count for U87MG Cells (Column I), LN18 Cells (Column II) and U118-MG Cells (Column III). The objective was to determine the relative effectiveness of TMZ, EPIGAL, TMQ and STAURO as apoptotic inducing agents.

MitoTracker Deep Red 633 is a membrane potential-dependent fluorescent dye that becomes permanently bound to the mitochondria and remains attached after the cell dies or is fixed. Cells were harvested and labeled with MitoTracker dye and analyzed by flow cytometry as

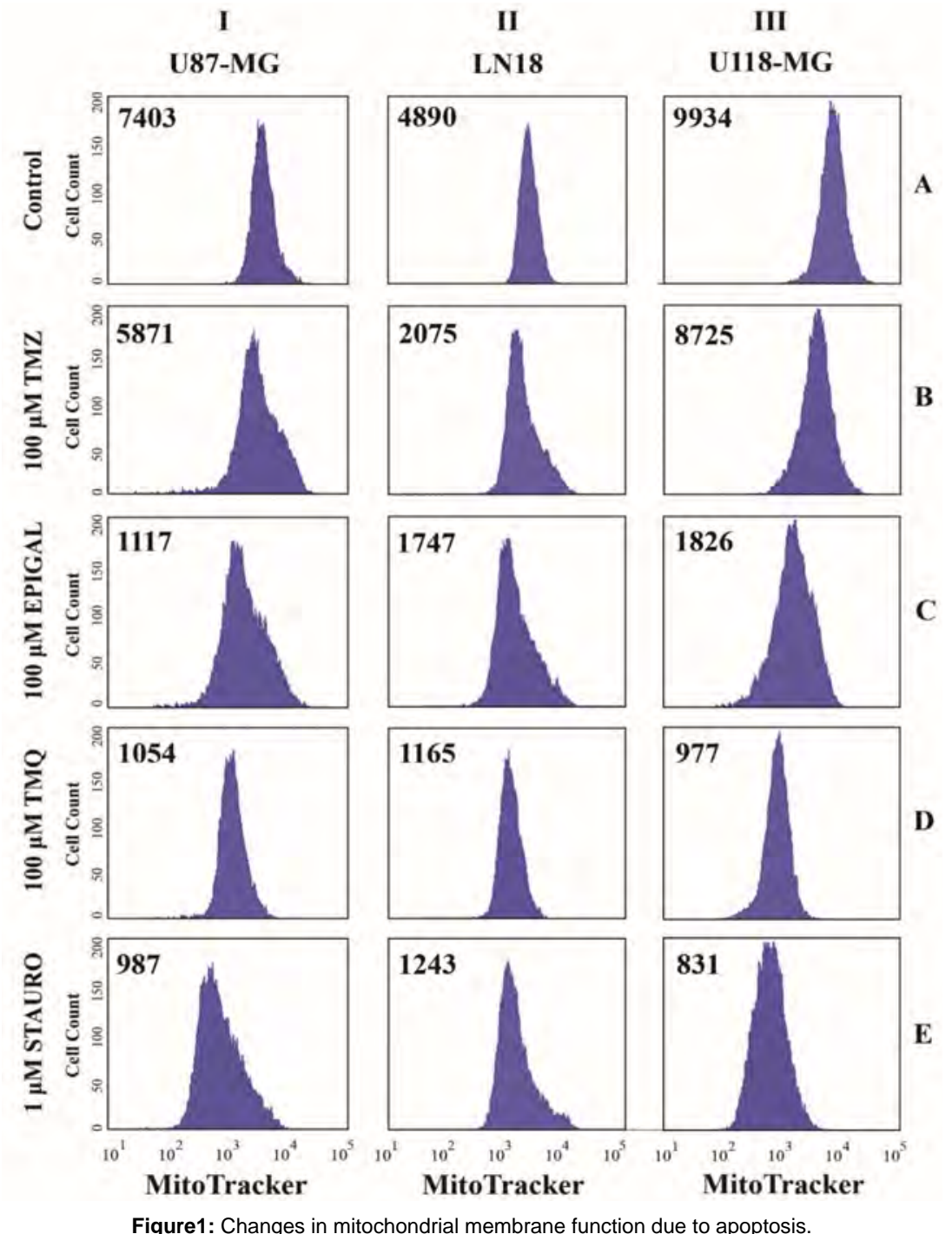


Citation: Magro A, Magro A, Shrestha S, Brundage K (2018) Apoptotic Effects of Temozolomide and Naturopathic Agents upon Glioblastoma Cells. J Oncol Transl Res 4: 132. doi: 10.4172/2476-2261.1000132

Page 4 of 10

outlined in the Methods. The DMSO vehicle controls of Figure 1 are represented by the row of panels labeled (A) while the TMZ, EPIGAL, TMQ and STAURO treated cells are represented by the rows of panels labeled (B), (C), (D) and (E) respectively. A decrease in fluorescence intensity indicates a decrease in mitochondrial membrane function, which is a telltale sign that apoptosis is occurring via a mitochondrial pathway. Changes in mitochondrial function are early events in the pharmacological induction of apoptosis. Downstream events of mitochondrial pore activation lead to programmed cell death.

The mean fluorescent Intensity (MFI) values of the vehicle control for the U87-MG, LN18 and U118-MG cells are shown to be 7403, 4890 and 9934 respectively. The treated cells shown in Figure 1 were acted upon with either $100 \mu \mathrm{M}$ TMZ, $100 \mu \mathrm{M}$ EPIGAL, $100 \mu \mathrm{M}$ TMQ, or 1 $\mu \mathrm{M}$ STAURO on a monolayer of the indicated glioblastoma cell lines for $8 \mathrm{~h}$ in 24 well plates. To varying degrees, the MFI of the emissions of the mitochondria bound MitoTracker Red decreases for all of the agents tested. The greatest down shifts in MFI were due to treatment of the 3 cell types with $1 \mu \mathrm{M}$ STAURO (panels of Row E) showing values 987,1243 and 831 , which calculate to be $74 \%, 87 \%$ and $92 \%$ decreases for the LN18, U87-MG and U118-MG cells respectively. The $100 \mu \mathrm{M}$ TMQ treated cells (panels of Row D) also show a significant downshift in the MFI for all the three cell types. The $100 \mu \mathrm{M}$ EPIGAL treated cells (panels of Row C) show less of a downshift, while the smallest down shifts of MitoTracker MFI were shown by the treatment of the cells with $100 \mu \mathrm{M}$ TMZ yielding decreases of $12 \%, 21 \%$ and $57 \%$ for the U118MG, U87-MG, LN18 and cells respectively. Taken as a whole, the data of Figure 1 indicate that TMZ is the least effective apoptotic inducing agent with STAURO being the most effective apoptotic inducing agent as measured by changes in mitochondrial function when tested upon the U87-MG, LN18 and U118-MG cell lines in vitro.

\section{Changes in cell populations of dot plots as an indicator of apoptosis}

Figure 2 consists of dot plots for glioblastoma cells labeled with

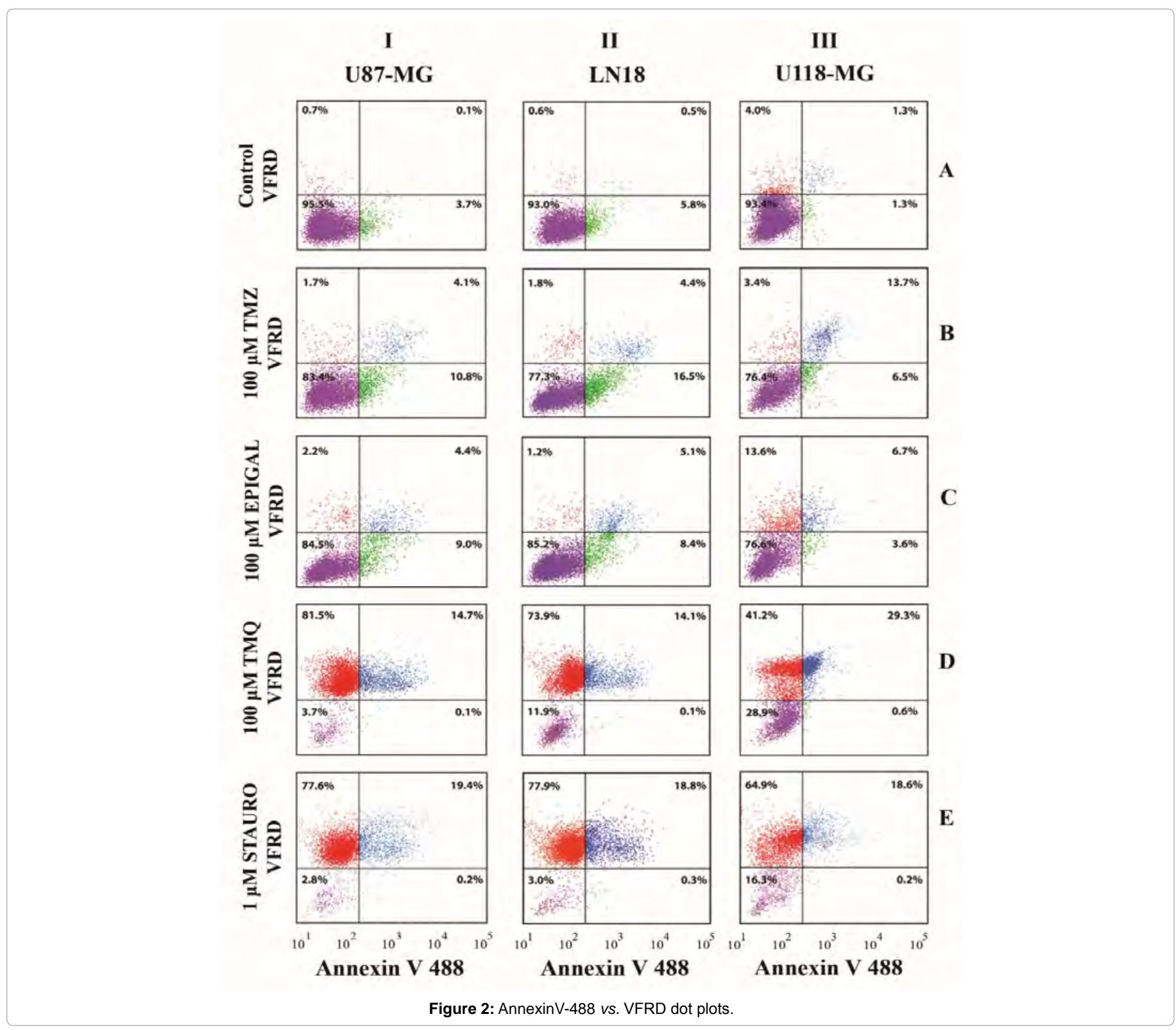


Citation: Magro A, Magro A, Shrestha S, Brundage K (2018) Apoptotic Effects of Temozolomide and Naturopathic Agents upon Glioblastoma Cells. J Oncol Transl Res 4: 132. doi: 10.4172/2476-2261.1000132

AnnexinV-488 and VFRD that were analyzed by flow cytometry. The objective was to determine the relative effectiveness of TMZ, EPIGAL, TMQ and STAURO as apoptotic inducing agents.

As described in the Methods, the 24 well plates consisting of a monolayer of glioblastoma cells were allowed to incubate for $12 \mathrm{~h}$ after the addition of the inducing agents. Detection of exposure of phosphatidylserine on the outer leaflet of the plasma membrane, which binds the fluorescent dye AnnexinV-488 is one of the classic tests for early and mid-apoptosis. Compromise of the plasma membrane allowing the amine binding dye Violet Fluorescent Reactive Dye (VFRD) to enter the cells is a classical test for mid and late apoptosis. Viable cells are not positive for AnnexinV-488 or VFRD and are neither apoptotic nor necrotic and are represented in the lower left quadrants of each panel; cells in early apoptosis stain for AnnexinV 488, but not VFRD and are in the lower right quadrants of each panel; cells that are in mid to late apoptosis are stained for both AnnexinV-488 and VFRD and are represented in the upper right quadrants of the panels; and cells in very late apoptosis stain positively for VFRD, but not for AnnexinV-488 and are represented in the upper left quadrants of each panel.

The numbers within the quadrants indicate the percentage of cells in each of the quadrants. The apoptotic inducing agents were $100 \mu \mathrm{M}$ TMZ, $100 \mu \mathrm{M}$ EPIGAL and $100 \mu \mathrm{M}$ TMQ illustrated in the panels of Rows (B), (C) and (D) respectively and $1 \mu \mathrm{M}$ STAURO shown in the panels of Row E. The non-treated DMSO vehicle control cells are shown in panels of Row A. The apoptotic agents are acting upon U87-MG cells (Column I), LN18 cells (Column II) and U118-MG cells (Column III).

Row (A) of Figure 2 presents the data for the DMSO vehicle controls with greater than $93 \%$ of the cells being in the lower left quadrant of the panels for all three cell types (Columns I, II and III) showing low PS exposure and low permeability to the dye VFRD indicating very low levels of apoptosis. The upper left quadrants of the three control panels (Row A) being less than $4 \%$ indicate that very few of the control cells are in a late apoptotic or necrotic state.

The TMZ treated cells in Row (B) of Figure 2 show that for all three cell types the majority of the cells are in the lower left quadrants (greater than $75 \%$ ) with less than $5 \%$ of the cells in late apoptosis (upper left quadrants of the panels) with a small population of cells in early and mid-apoptosis (lower right and upper right quadrants of the panels). Thus, following $12 \mathrm{~h}$ treatment with $100 \mu \mathrm{M}$ of TMZ a small percentage of the cells are in late apoptosis with less than $22 \%$ of the cells being in early to mid-apoptosis.

The data of Row $\mathrm{C}$ show that EPIGAL has a very similar dot pattern to TMZ (Row B) for the cell types U87-MG (Column I) and LN18 (Column II) with a slightly higher percentage of cells in the upper left quadrant (13.6\%) for the U118-MG cells (Column III). Overall, the dot plots of Row (B) and (C) show that TMZ and EPIGAL are weak apoptotic agents when acting upon a monolayer of U87-MG, LN18 and U118-MG cell lines for $12 \mathrm{~h}$.

The data of Rows (D) and (E) of Figure 2 show that $100 \mu \mathrm{M}$ TMQ and $1 \mu \mathrm{M}$ STAURO are effective apoptotic inducing agents when acting on the U87-MG, LN18 and U118-MG cell lines for $12 \mathrm{~h}$ in a monolayer. This is manifested by a high population of cells in mid and late apoptosis (upper right and upper left quadrants) with a lower population of cells in non-apoptotic and early apoptotic states (lower left and lower right quadrants). In total, the dot plot data indicate that in all three of the cell lines TMZ at $100 \mu \mathrm{M}$ was mildly effective as an apoptotic inducing agent, while STAURO at $1 \mu \mathrm{M}$ was an effective apoptotic-inducing agent. Likewise, EPIGAL at $100 \mu \mathrm{M}$ was relatively ineffective (Row C) as an apoptotic-inducing agent as compared to the TMQ and STAURO (Rows D and E respectively).

\section{Effects of the apoptotic inducing agents upon glioblastoma invasiveness in the $3 \mathrm{D}$ matrigel spheroidal assay}

Figures 3 to 5 are micrographs of glioblastoma cell lines U87-MG, LN18 and U118-MG that illustrate 3D invasiveness emanating from spheroids embedded in a collagen enriched matrigel [51]. The objective was to determine if the apoptotic inducing agents TMZ, EPIGAL, TMQ and STAURO have the ability to inhibit glioblastoma cell invasiveness. The data of Figures 3 to 5 are representative of a minimum of three spheroidal invasiveness experiments.

Three dimensional spheroidal cell cultures are better approximates of the in vivo conditions surrounding solid tumors. Micrographs illustrating spheroid formation of Day 1 resulted from an initial seeding of 6,000 cells. Micrographs were acquired at 40x with a Nikon Diaphot inverted microscope equipped with Prog Res Capture imaging software and camera. Micrographs were taken on Day 1 and Day 5 . The micrographs include the untreated control and $100 \mu \mathrm{M} \mathrm{TMZ}, 100 \mu \mathrm{M}$ TMQ, $100 \mu \mathrm{M}$ EPIGAL and $1 \mu \mathrm{M}$ STAURO treated cells. As mentioned in the Methods, the areas of the spheroid images for each micrograph

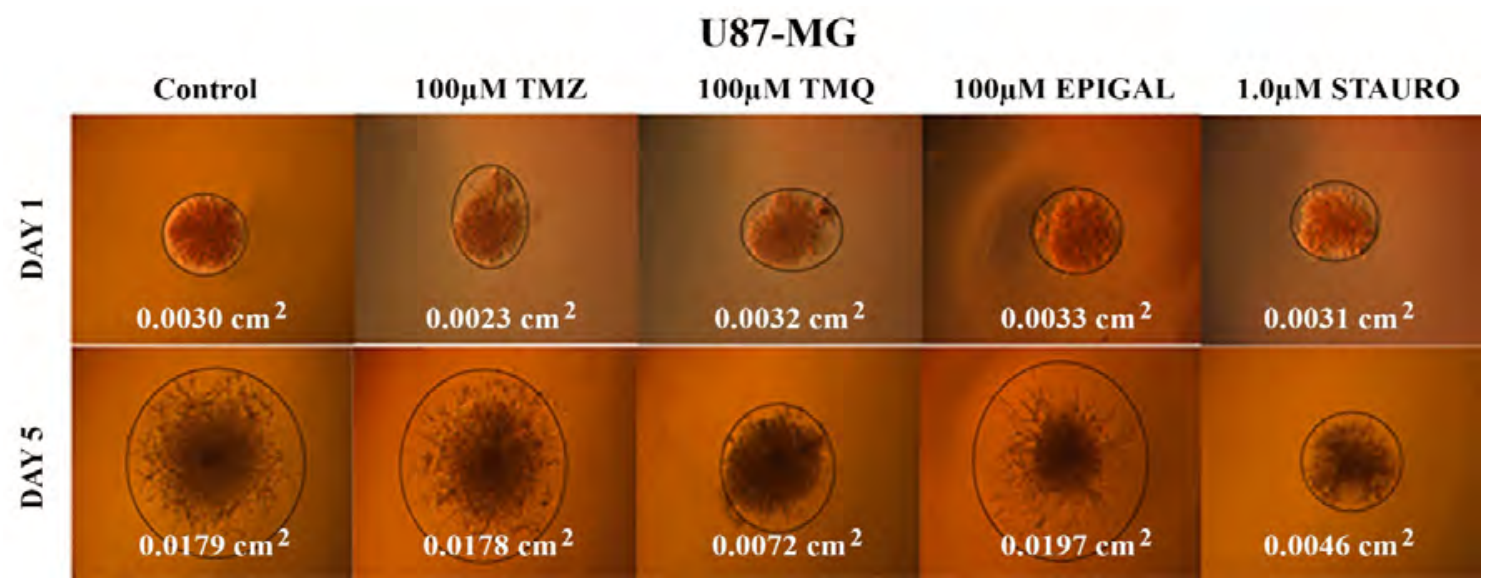

Figure 3: Invasiveness of U87-MG Cell Line on Day 1 and Day 5. 
Citation: Magro A, Magro A, Shrestha S, Brundage K (2018) Apoptotic Effects of Temozolomide and Naturopathic Agents upon Glioblastoma Cells. J Oncol Transl Res 4: 132. doi: 10.4172/2476-2261.1000132

\section{LN18}

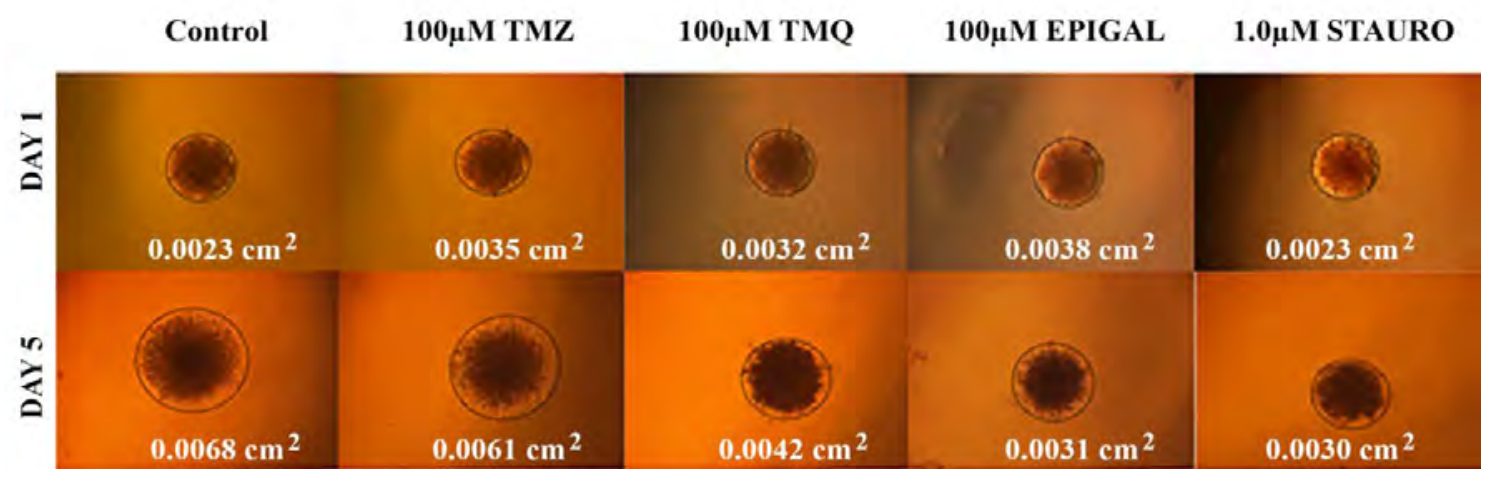

Figure 4: Invasiveness of LN18 Cell Line on Day 1 and Day 5.

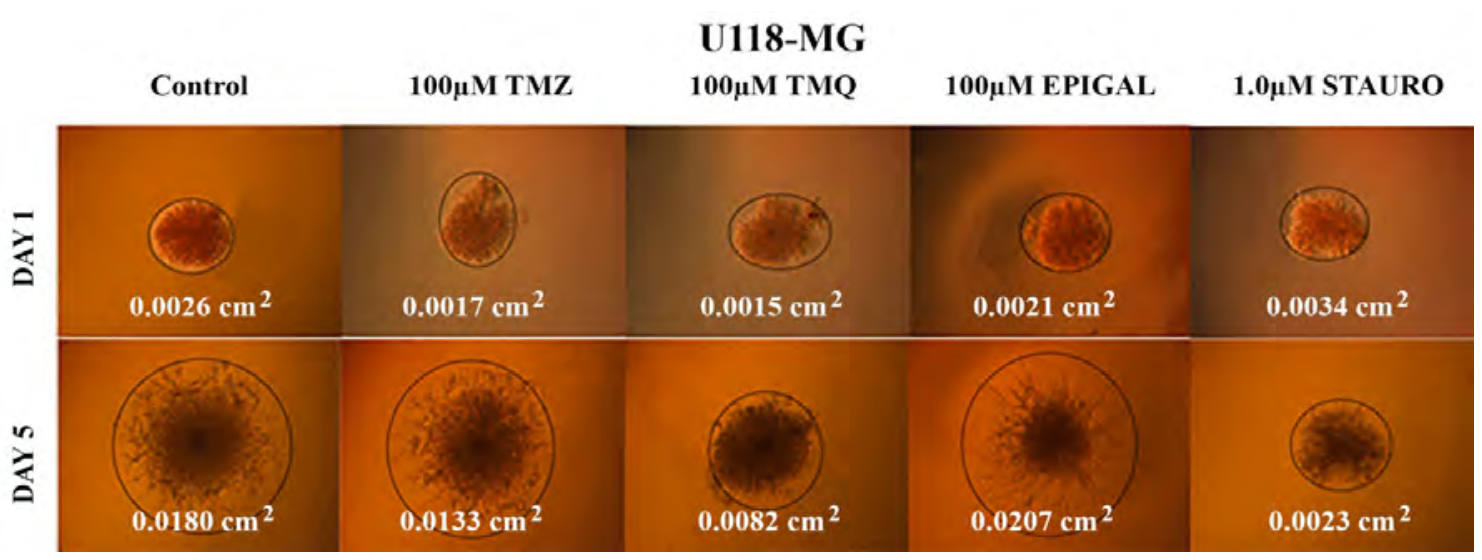

Figure 5: Invasiveness of U118-MG Cell Line on Day 1 and Day 5.

were calculated using Image J software and are located at the bottom of the respective micrographs. The values of the spheroid areas are shown within the micrographs.

The formation of polarized extensions, such as invadopodia emanating from cells within a solid tumor is a hallmark of infiltration through a $3 \mathrm{D}$ environment [52]. These protrusive structures formed by migrating and invading cells appear on the leading edges of migrating cells and function to command the direction of the migrating cells. Invadopodia are special F-actin-rich matrix-degrading structures that arise on the surface of the cell membrane. When comparing the control micrographs of Day 1 to the control micrographs of Day 5 , all cell types showed an increase in growth and invasiveness over the 5 days.

Figure 3 illustrates how the invasiveness of the U87-MG cell line is affected by $100 \mu \mathrm{M}$ TMZ, $100 \mu \mathrm{M}$ TMQ, $100 \mu \mathrm{M}$ EPIGAL and 1 $\mu \mathrm{M}$ STAURO. First, looking at the non-treated control (column 1) of the U87-MG cell line it can be seen that the spheroid within the micrograph of Day 5 (row 2 of column 1) is more than 5 times larger in area than that of the spheroid within the micrograph of Dayl (row 1 of column 1). In addition to its dimensional growth, the spheroid of the 5-day control shows long extensive filopodia indicating that the U87MG cells are highly invasive. The spheroids of the Day 5 micrographs of Figure 3 for $100 \mu \mathrm{M}$ TMZ (row 2 of column 2) and $100 \mu \mathrm{M}$ EPIGAL (row 2 of column 4) as compared to the spheroid of the Day 5 control cells (row 2 of column 1) show little difference indicating that TMZ and EPIGAL are not effective inhibitors of invasiveness when acting upon the U87-MG cell line. Comparing the filopodia lengths and areas of the Day 5 spheroids within the micrographs of $100 \mu \mathrm{M}$ TMQ (row 2, column 3) and $1 \mu \mathrm{M}$ STAURO (row 2, column 5) to the Day 5 control (row 2 of column 1) it can be seen that TMQ and STAURO are effective inhibitors of spheroidal invasiveness.

The spheroidal area within the micrographs of Figure 4 show the control LN18 cells (column 1 of Figure 4) increased 3 fold over the 5 days (column 1, row 2) and manifest short filopodia like extensions emanating away from the outer edge of the spheroid. The spheroid of the Day 5 (column 2, row 2) micrograph of $100 \mu \mathrm{M} \mathrm{TMZ}$ manifests filopodia and an area similar to that of Day 5 control (column 1, row 2) indicating that TMZ is not an effective inhibitor of invasiveness of the LN18 cell line. The Day 5 micrographs of $100 \mu \mathrm{M}$ TMQ (column 3, row 2), $100 \mu \mathrm{M}$ EPIGAL (column 4, row 2) and $1 \mu \mathrm{M}$ STAURO (column 5 , row 2), when compared to the Day 5 control (column 1, row 2), indicate that TMQ, EPIGAL and STAURO have the ability to inhibit invasiveness of the LN18 cells line.

Figure 5 illustrates how invasiveness of the U118-MG cell line is affected by the apoptotic inducing agents. The invasiveness of the nontreated control cells were compared to cells treated with $100 \mu \mathrm{M} \mathrm{TMZ}$, $100 \mu \mathrm{M}$ TMQ, $100 \mu \mathrm{M}$ EPIGAL and $1 \mu \mathrm{M}$ STAURO. Looking at the 
Citation: Magro A, Magro A, Shrestha S, Brundage K (2018) Apoptotic Effects of Temozolomide and Naturopathic Agents upon Glioblastoma Cells. J Oncol Transl Res 4: 132. doi: 10.4172/2476-2261.1000132

Page 7 of 10

non-apoptotic control micrographs of the U118-MG cell line it can be seen that the spheroid within the micrograph of Day 5 (row 2 of column 1) is more than 5 times larger in area than that of the spheroid within the micrograph of Day 1 (row 1 of column 1). The spheroid of the 5-day control shows long extensive filopodia indicating that the U118-MG cells are highly invasive. The spheroids of the Day 5 micrographs for $100 \mu \mathrm{M}$ TMZ (row 2 of column 2) as compared to the Day 5 spheroid of the control cells (row 2 of column 1) shows that TMZ is not an effective inhibitor of invasiveness when acting upon the U118-MG cell line. The Day 5 spheroid of the U118-MG cells treated with $100 \mu \mathrm{M}$ EPIGAL (row 2 of column 4) is actually larger than the U118-MG Day 5 control (row 5 of column 1). Comparing the filopodia lengths and areas of the spheroids within the Day 5 micrographs of $100 \mu \mathrm{M}$ TMQ (row 2 of column 3 ) and $1 \mu \mathrm{M}$ STAURO (row 2 of column 5) shows significant inhibition of invasiveness when compared to the Day 5 control (row 2 of column 1). In comparing their abilities to inhibit invasiveness TMZ, EPIGAL, TMQ and STAURO had similar overall effects upon the U87MG cells (Figure 3) and the U118-MG cells (Figure 5).

Effect of apoptotic inducing agents upon the degradation of class I histocompatibilty cell-surface determinants

The histograms of Figure 6 are plots of HLA Class I expression versus cell count. The intent was to demonstrate that the U87-MG, LN18 and U118-MG cell lines have demonstrable levels of Class I

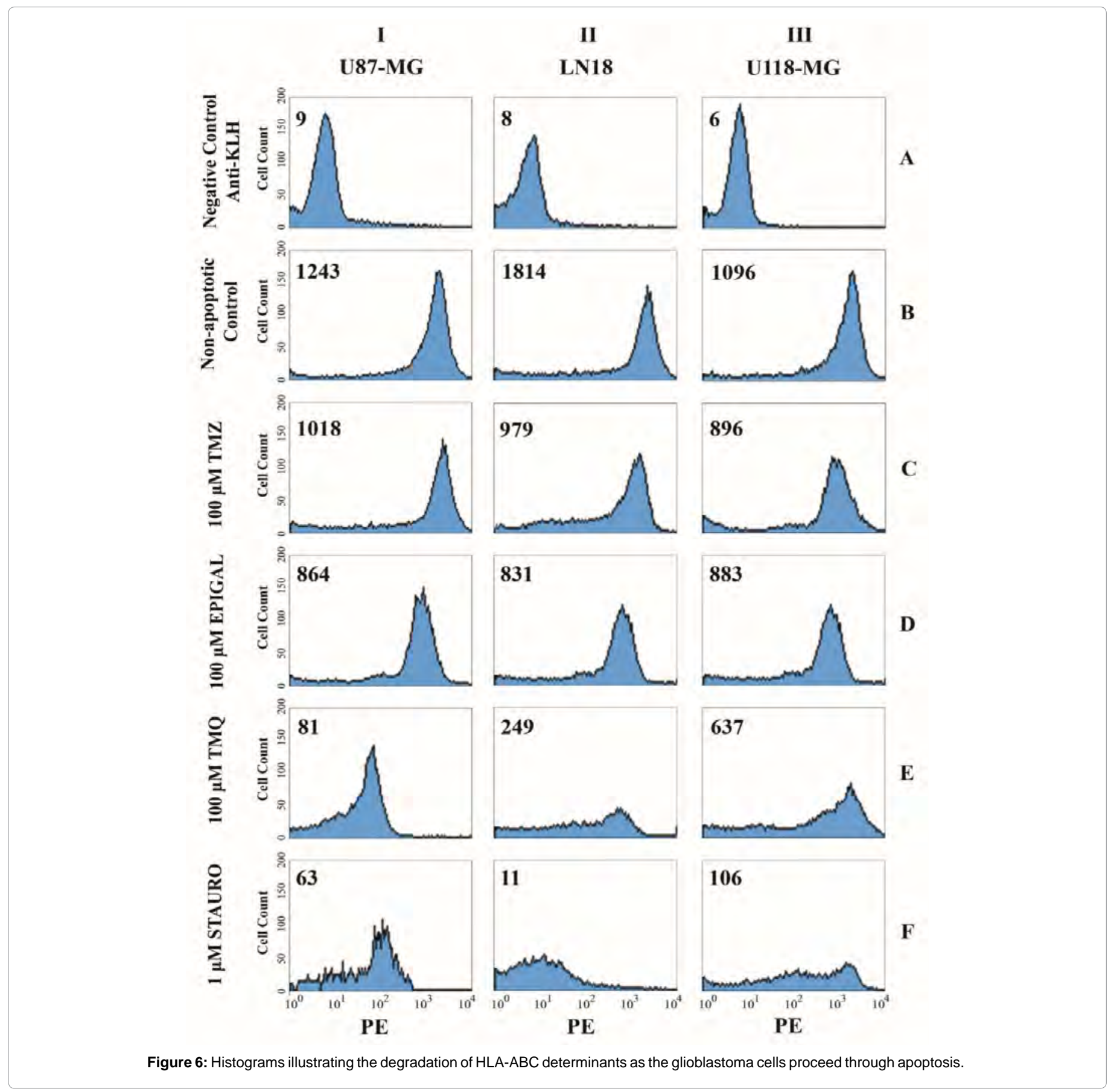


histocompatibilty leukocyte antigens (HLA-ABC) and to determine the degradation effects of apoptotic inducing agents upon the class I histocompatibilty antigens. The values within the panels of Figure 6 indicate the detected values of the phycoerythrin mean fluorescent intensity (MFI) emanating from the surface of the glioblastoma cells for apoptotic and non-apoptotic glioblastoma cells. The determinants on the surface of the glioblastoma cells were detected by mouse antiHLA-ABC as primary antibody. For all of the histograms of Figure 6 the secondary antibody used was $\mathrm{F}(\mathrm{ab})_{2}$-goat anti-rabbit IgG conjugated to the fluorescent phycoerythrin. A low MFI indicates a low density of the class 1 determinants on the surface of the glioblastoma cells.

In Figure 6 Rabbit anti-keyhole limpet hemocyanin (anti-KLH) was used as a non-specific primary rabbit antibody control. The nonbinding rabbit anti-KLH controls (Row A) show low MFI values for the U87-MG (Column I), LN18 (Column II) and U118-MG (Column III) cell lines indicating very low binding for the non-specific rabbit antibody for all three-cell types. For Rows B through F rabbit antiHLA-ABC was used as a primary antibody. Row (B) of Figure 6 shows the MFI for the non-apoptotic controls. The high MFI values within the panels of Row B indicate that the glioblastoma cell lines U87-MG, LN18 and U118-MG in a non-apoptotic state with MFI values of 1243, 1814 and 1096 respectively, have high levels of cell surface Class I histocompatibilty antigens.

The data of Figure 6 for Rows (C) to Row (F) illustrate the degradation of the histocompatibility class I determinants as all three glioblastoma cell types proceed through apoptosis. Apoptosis was induced by TMZ (Row C), EPIGAL (Row D). TMQ (Row E) and STAURO (Row F). Examining the MFIs of TMZ treated cells (Row C) compared to the non-apoptotic controls (Row B) it can be seen that $100 \mu \mathrm{M}$ TMZ does have a slight ability to induce the degradation of Class I histocompatibility determinants with MFI values of 1018, 979 and 896 which calculate to be $18 \%, 18 \%$ and $46 \%$ decreases for the U87-MG, U118-MG and LN18 cells respectively. The losses in class I determinants of the EPIGAL treated cells (Row D) are similar to the TMZ treated cells, but the losses of class I determinants were more complete for the TMQ treated cells (Row E) and the STAURO treated cells (Row F). The TMQ treated cells show decreases of $42 \%$, $86 \%$ and $93 \%$ while the STAURO treated cells show deceases of $95 \%$, $98 \%$ and $99 \%$ for the U87-MG, LN18 and U118-MG cells respectively. Comparison the data of Figures 1 and 2 to the data of Figure 6 indicates that the apoptotic effectiveness of the inducing agent is proportional to the apoptotic agent's ability to promote degradation of the cell-surface class I MHC determinants.

\section{Discussion}

The treatment of tumors by irradiation or chemotherapeutic agents induces apoptosis. However, within the population of the treated tumors there is invariably a mixture of resistant non-apoptotic cells along with a mixture of cells in different stages of apoptosis [53]. Chemotherapeutic treated glioblastoma multiformes (GBMs) and other high-grade gliomas can readily mutate into therapeutic resistant forms [54] leaving only the defenses of acquired and natural immune responses. A known feature of glioblastoma multiformes (GBMs) is that they are immunosuppressive [55]. Surface cluster determinants are important stimulants of natural immune mechanisms with class I major histocompatibility complexes (MHC) being particularly important to acquired immune defenses against tumors [56]. Apoptotic cells secrete pericellular metalloproteinases and serine proteases capable of degrading cell surface determinants of the apoptotic cells themselves and determinants of the surrounding non-apoptotic cells within the tumor [46]. To escape immune surveillance, tumor cells have the innate ability to diminish cell surface determinants that stimulate natural and acquired immune mechanisms [57]. Within a tumor, the degradation of immune stimulating surface determinants of neighboring cells by the metalloproteinases and serine proteases secreted from cells that are in late apoptosis can contribute to the tumor's ability to circumvent natural and acquired immune defenses.

In vitro testing of the ability to induce rapid and acute apoptosis or to inhibit invasiveness may not be the best predictors of the in vivo efficacy of a chemotherapeutic drug. Temozolomide (TMZ) being the drug of choice in the treatment of GBMs was not the most effective apoptotic inducing agent nor was it the most effective in its inhibition of invasiveness. The in vitro mitochondrial function and dot plot tests performed here show that TMZ was not rapid as an apoptotic-inducing agent. The in vitro dot plots illustrated that TMZ treated glioblastoma cells had a small population of cells in late apoptosis. Also, by the 3D spheroid test it was determined that the TMZ did not effectively inhibit invasiveness. The data raise the question as to why $\mathrm{TMZ}$ is effective as an adjuvant drug in the treatment of GBM. Overall, the data presented here indicate that there may be in vivo effects other than those manifested by in vitro apoptotic and spheroidal testing that are more relevant with regard to TMZ's clinical effectiveness. Also, in comparing the apoptotic and anti-invasive properties of the naturopathic agents thymoquinone (TMQ) and epigallocatechin gallate (EPIGAL), one would expect TMQ to be a more promising antineoplastic agent than EPIGAL in that TMQ showed a greater ability to induce apoptosis and to inhibit invasiveness of the glioblastoma cell lines. However, EPIGAL, a well-tolerated extract from tea, does manifest benefits in the treatment of malignant tumors [24-28] implying that molecules related to EPIGAL cannot be discounted as having the potential to be well-tolerated anti-neoplastic agents.

The antineoplastic mechanisms of TMZ are quite complex [58]. TMZ is spontaneously hydrolyzed to the active compound 5-(3-dimethyl-1-triazenyl) imidazole-4-carboxamide (MTIC) which is capable delivering a methyl group to purine bases of DNA (O6guanine; N7-guanine and N3-adenine) causing base pair mismatches that can arrest the cells in various stages of mitosis and eventually trigger cytotoxicity and apoptosis [59]. Tumor cells possess mismatchrepair mechanisms such as $\mathrm{O}^{6}$-methylguanine-DNA methyltransferase (MGMT), which is a DNA-repair protein that removes methyl groups from the $\mathrm{O}^{6}$ position of guanine [60]. It is likely that there are additional repair mechanisms yet to be identified. TMZ is rendered more effective when administered in repetitive doses at a high frequency [61]. It is also rendered more effective when administered with an agent that inhibits the methyltransferase action of MGMT [62]. TMZ does not require enzymatic conversion in the liver to the active component MTIC [63] and both MTIC and unconverted TMZ pass the blood brain barrier, which may be a factor as to why TMZ is more effective in the treatment of GBMs than carmustine and other alkylating agents. However, a possibility that is raised here is that the clinical effectiveness of TMZ may be in part related to it being less toxic and less acute in its apoptotic properties. Stated more explicitly, if GBM cells treated with TMZ are arrested sufficiently and slowly progress through apoptosis and do not affect the immune stimulating capacity of surrounding cells within the GBM tumor, it may permit immune surveillance mechanisms to remain intact longer.

The many difficulties in developing a successful pharmaceutical agent are well known which include being well tolerated with few 
Citation: Magro A, Magro A, Shrestha S, Brundage K (2018) Apoptotic Effects of Temozolomide and Naturopathic Agents upon Glioblastoma Cells. J Oncol Transl Res 4: 132. doi: 10.4172/2476-2261.1000132

contraindications. These and other concerns are standard considerations in drug development that pharmaceutical houses pay great attention to. They are mentioned here to call attention to the fact that in drug development the question of whether chemotherapeutic agents have immunosuppressive effects has been somewhat neglected. Apoptotic glioblastoma cells have very different characteristics than nonapoptotic glioblastoma cells. The ability to induce acute apoptosis may not be the best predictor of a clinically effective anti-neoplastic drug. What is proposed here is that cells in late apoptosis can exacerbate the immune suppression of glioblastoma patients and may interfere with the sustained effectiveness of natural and acquired immune defenses.

\section{Acknowledgement}

Research reported in this publication was supported by the National Institute of General Medical Sciences of the National Institutes of Health under Award Number P20GM103434. The content is solely the responsibility of the authors and does not necessarily represent the official views of the National Institutes of Health. Flow cytometry experiments were performed in the West Virginia University Flow Cytometry and Single Cell Core Facility, which is supported by NIH grants P20GM103488 and S100DO16165.

\section{References}

1. Surawicz TS, Davis F, Freels S, Laws Jr ER, Menck HR (1998) Brain tumor survival: Results from the national cancer data base. J Neuro-Oncol 40: 151160

2. Kanu OO, Mehta A, Di C, Lin N, Bortoff K, et al. (2009) Glioblastoma multiforme: A review of therapeutic targets. Expert Opin Ther Targets 13: 701-718.

3. Salvati M, D'Elia A, Formichella Al, Frati A (2009) Insights into pharmacotherapy of malignant glioma in adults. Expert Opin Pharmacother 10: 2279-2290.

4. Minniti G, Muni R, Lanzetta G, Marchetti P, Enrici RM (2009) Chemotherapy for glioblastoma: Current treatment and future perspectives for cytotoxic and targeted agents. Anticancer Res 12: 5171-5184.

5. Stupp R, Mason WP, van den Bent MJ, Weller M, Fisher B, et al. (2005) Radiotherapy plus concomitant and adjuvant temozolomide for glioblastoma. N Engl J Med 352: 987-996.

6. Lo HW (2010) EGFR-targeted therapy in malignant glioma: Novel aspects and mechanisms of drug resistance. Curr Mol Pharmacol 3: 37-52.

7. Salacz ME, Watson KR, Schomas DA (2011) Glioblastoma. Part II: Future directions. Mo Med 108: 289-291.

8. Jain A, Lai JCK, Chowdhury GMI, Behar K, Bhushan A (2011) Glioblastoma: Current chemotherapeutic status and need for new targets and approaches.

9. Giese A, Bjerkvig R, Berens ME, Westphal M (2003) Cost of migration: Invasion of malignant gliomas and implications for treatment. J Clin Oncol 21: 16241636.

10. Chen C, Xu T, Lu Y, Chen J, Wu S (2013) The efficacy of temozolomide for recurrent glioblastoma multiforme. Eur J Neurol 20: 223-230.

11. Wick W, Platten M, Meisner C, Felsberg J, Tabatabi G, et al. (2012) Temozolomide chemotherapy alone versus radiotherapy alone for malignant astrocytoma in the elderly: The NOA-08 randomised, phase 3 trial. Lancet Oncol 13: 707-715.

12. Malmstrom A, Gronberg BH, Marosi C, Stupp R, Frappaz D, et al. (2012) Temozolomide versus standard 6-week radiotherapy versus hypofractionated radiotherapy in patients older than 60 years with glioblastoma: The Nordic randomised, phase 3 trial.m. Lancet Oncol 209: 916-926.

13. Fukushima T, Takeshima $H$, Kataoka $H$ (2009) Anti-glioma therapy with temozolomide and status of the DNA-repair gene MGMT. Anticancer Res 29 : 4845-4854.

14. Quinn JA, Jiang SX, Reardon DA, Desjardins A, Vredenburgh JJ, et al. (2009) Phase II trial of temozolomide plus O6-benzylguanine in adults with recurrent, temozolomide-resistant malignant glioma. J Clin Oncol 27: 1262-1267.

15. Balmaceda C, Peereboom D, Pannullo S, Cheung YK, Fisher PG, et al. (2008) Multi-institutional phase II study of temozolomide administered twice daily in the treatment of recurrent high-grade gliomas. Cancer 112: 1139-1146.

16. Fountzilas G, Karkavelas G, Kalogera-Fountzila A, Karina M, Ignatiadis $\mathrm{M}$, et al. (2006) Postoperative combined radiation and chemotherapy with temozolomide and irinotecan in patients with high-grade astrocytic tumors. A phase II study with biomarker evaluation. Anticancer Res 26: 4675-4686.

17. Brandes AA, Tosoni A, Cavallo G, Bertorelle R, Gioia V, et al. (2006) GICNO Temozolomide 3 weeks on and 1 week off as first-line therapy for recurrent glioblastoma: Phase II study from Gruppo Italiano Cooperativo di Neurooncologia (GICNO). Br J Cancer 95: 1155-1160.

18. Belmokhtar CA, Hillion J, Ségal-Bendirdjian E (2001) Staurosporine induces apoptosis through both caspase-dependent and caspase-independent mechanisms. Oncogene 20: 3354-3362.

19. De Witt Hamer PC (2010) Small molecule kinase inhibitors in glioblastoma: A systematic review of clinical studies. Neuro-Oncol 12: 304-316.

20. Attoub S, Sperandio O, Raza H, Arafat K, Al-Salam S, et al. (2013) Thymoquinone as an anticancer agent: evidence from inhibition of cancer cells viability and invasion in vitro and tumor growth in vivo. Fundam Clin Pharmacol 27: 557-569.

21. Lei X, Lv X, Liu M, Yang Z, Ji M, et al. (2012) Thymoquinone inhibits growth and augments 5-fluorouracil-induced apoptosis in gastric cancer cells both in vitro and in vivo. Biochem Biophysics Res Commun 417: 864-868.

22. Woo CC, Loo SY, Gee V, Yap CW, Sethi G, et al. (2011) Anticancer activity of thymoquinone in breast cancer cells: Possible involvement of PPAR-pathway. Biochem Pharmacol 82: 464-475.

23. El-Najjar N, Chatila M, Moukadem H, Vuorela H, Ocker M, et al. (2010) Reactive oxygen species mediate thymoquinone-induced apoptosis and activate ERK and JNK signaling. Apoptosis 15: 183-195.

24. Chen D, Pamu S, Cui Q, Chan TH, Dou QP (2012) Novel epigallocatechin gallate (EGCG) analogs activate AMP-activated protein kinase pathway and target cancer stem cells. Bioorg Med Chem 20: 3031-3037.

25. Shim JH, Su ZY, Chae JI, Kim DJ, Zhu F, et al. (2010) Epigallocatechin gallate suppresses lung cancer cell growth through ras-GTPase-activating protein SH3 domain-binding protein 1. Cancer Prev Res 3: 670-679.

26. Shammas MA, Neri P, Koley H, Batchu RB, Bertheau RC, et al. (2006) Specific killing of multiple myeloma cells by (-)-epigallocatechin-3-gallate extracted from green tea: Biologic activity and therapeutic implications. Blood 108: 2804-2810.

27. Fang MZ, Want Y, Ai N, Hou Z, Sun Y, et al. (2003) Tea polypheno (-)-epigallocatechin-3-gallate inhibits DNA methyltransferase and reactivates methylation-silenced genes in cancer cell lines. Cancer Res 63: 7563-7570.

28. Lambert JD, Yang CS (2003) Mechanisms of cancer prevention by tea constituents. J Nutr 133: 3262S-3267S.

29. Yadav SS, Prasad CB, Prasad SB, Pandey LK, Singh S, et al. (2015) Anti-tumor activity of staurosporine in the tumor microenvironment of cervical cancer: An in vitro study. Life Sci 133: 21-28.

30. Simenc J, Lipnik-Stangelj M (2012) Staurosporine induces different cell death forms in cultured rat astrocytes. Radiol Oncol 46: 312-320.

31. El-Mahdy MA, Zhu Q, Wang QE, Wani G, Wani AA, et al. (2005) Thymoquinone induces apoptosis through activation of caspase-8 and mitochondrial events in p53-null myeloblastic leukemia HL-60 cells. Int J Cancer 117: 409-417.

32. Shoieb AM, Elgayyar M, Dudrick PS, Bell JL, Tithof PK (2003) In vitro inhibition of growth and induction of apoptosis in cancer cell lines by thymoquinone. Int J Oncol 22: 107-113.

33. Shankar S, Suthakar G, Srivastava RK (2007) Epigallocatechin-3-gallate inhibits cell cycle and induces apoptosis in pancreatic cancer. Front Biosci 12 5039-5051.

34. Gupta S, Hastak K, Afaq F, Ahmad N, Mukhtar H (2004) Essential role of caspases in epigallocatechin-3-gallate-mediated inhibition of nuclear factor kappa B and induction of apoptosis. Oncogene 23: 2507-2522.

35. Yokoyama M, Noguchi M, Nakao Y, Pater A, Iwasaka T (2004) The tea polyphenol, (-)-epigallocatechin gallate effects on growth, apoptosis and telomerase activity in cervical cell lines. Gynecol Oncol 92: 197-204.

36. Borska S, Gebarowska E, Wysocka T, Drag-ZalesiÅska M, Zabel M (2003) Induction of apoptosis by EGCG in selected tumour cell lines in vitro. Folia Histochem Cytobiol 41: 229-232.

37. Alam S, Khan ZI, Mustafa G, Kumar M, Islam F, et al. (2012) Development and evaluation of thymoquinone-encapsulated chitosan nanoparticles for nose-to-brain targeting: A pharmacoscintigraphic study. Int J Nanomedicine 7: 5705-5718. 
Citation: Magro A, Magro A, Shrestha S, Brundage K (2018) Apoptotic Effects of Temozolomide and Naturopathic Agents upon Glioblastoma Cells. J Oncol Transl Res 4: 132. doi: 10.4172/2476-2261.1000132

Page 10 of 10

38. Sheikh BY, Mohamadin AM (2012) Thymoquinone a potential therapy for cerebral oxidative stress. Asian J Nat \& Appl Sci 1: 176-182.

39. Hosseinzadeh $\mathrm{H} 1$, Parvardeh S (2004) Anticonvulsant effects of thymoquinone, the major constituent of Nigella sativa seeds, in mice. Phytomedicine 11: 56-64.

40. Lin LC1, Wang MN, Tseng TY, Sung JS, Tsai TH (2007) Pharmacokinetics of (-)-epigallocatechin-3-gallate in conscious and freely moving rats and its brain regional distribution. J Agric Food Chem 55: 1517-1524.

41. Nath S, Bachani M, Harshavardhana D, Steiner JP (2012) Catechins protect neurons against mitochondrial toxins and HIV proteins via activation of the BDNF pathway. J Neurovirol 18: 445-455.

42. Park JW, Jang YH, Kim JM, Lee H, Park WK, et al. (2009) Green tea polyphenol (-)-epigallocatechin gallate reduces neuronal cell damage and up-regulation of MMP-9 activity in hippocampal CA1 and CA2 areas following transient global Cerebral ischemia. J Neurosci Res 87: 567-575.

43. Rao JS1 (2003) Molecular mechanisms of glioma invasiveness: the role of proteases. Nat Rev Cancer 3: 489-501.

44. Bubeník J (2004) MHC class I down-regulation: tumour escape from immune surveillance? (Review). Int J Oncol 25: 487-491.

45. Magro A, MagroAD, Shrestha S, Brundage K, Rankin G (2014) Metalloproteinase dependent reduction of cell surface cluster determinants upon the induction of apoptosis. Int J Oncol 44: 19-50.

46. Magro A, Magro A, Brundage K, Rankin G (2017) Apoptotic increases in pericellular protease activity and glioblastoma escape from immune surveillance in glioblastoma.

47. Diserens AC, de Tribolet N, Martin-Achard A, Gaide AC, Schnegg JF, et al (1981) Characterization of an established human malignant glioma cell line: LN-18. Acta Neuropathol 53: 21-28.

48. Pontén J, Macintyre EH (1968) Long term culture of normal and neoplastic human glia. Acta Pathol Microbiol Scand 74: 465-486.

49. Bullard DE, Schold SC Jr, Bigner SH, Bigner DD (1981) Growth and chemotherapeutic response in athymic mice of tumors arising from human glioma-derived cell lines. J Neuropathol Exp Neurol 40: 410-27.

50. Zhou Q, Lv H, Mazloom AR, Xu H, Ma'ayan A, et al. (2012) Activation of alternate prosurvival pathways accounts for acquired sunitinib resistance in U87MG glioma xenografts. J Pharmacol Exp Ther 343: 509-519.

51. Vinci M, Box C, Zimmermann M, Eccles SA (2013) Tumor spheroid-based migration assays for evaluation of therapeutic agents. Methods Mol Biol 986: 253-266.

52. Mallawaaratchy DM, Buckland ME, McDonald KL, Li CC, Ly L, et al. (2015) Membrane proteome analysis of glioblastoma cell invasion. J Neuropathol Exp Neurol 74: 425-441.

53. Fulda S (2009) Tumor resistance to apoptosis. Int J Cancer 124: 511-515.

54. Osuka S, Van Meir EG (2017) Overcoming therapeutic resistance in glioblastoma: The way forward. J Clin Invest 127: 415-426.

55. Vega EA, Graner MW, Sampson JH (2008) Combating immunosuppression in glioma. Future Oncol 4: 433-442.

56. Caron E, Vincent K, Fortier MH, Laverdure JP, Bramoullé A, et al. (2011) The $\mathrm{MHC}$ I immunopeptidome conveys to the cell surface an integrative view of cellular regulation. Mol Syst Biol 7: 533.

57. Kim R, Emi M, Tanabe K (2007) Cancer immuno editing from immune surveillance to immune escape. Immunology 121: 1-14.

58. Zhang J, Stevens MF, Bradshaw TD (2012) Temozolomide: Mechanisms of action, repair and resistance. Curr Mol Pharmacol 5: 102-114.

59. Noonan EM, Shah D, Yaffe MB, Lauffenburger DA, Samson LD (2012) O6Methylguanine DNA lesions induce an intra-S-phase arrest from which cells exit into apoptosis governed by early and late multi-pathway signaling network activation. Integr Biol (Camb) 4: 1237-1255.

60. Modrich P (2006) Mechanisms in eukaryotic mismatch repair. J Biol Chem 281 30305-30309.

61. Wick W, Platten M, Weller M (2009) New (alternative) temozolomide regimens for the treatment of glioma. Neuro Oncol 11: 69-79.

62. Hegi ME, Diserens AC, Gorlia T, Hamou MF, de Tribolet N, et al. (2005) MGMT gene silencing and benefit from temozolomide in glioblastoma. N Engl J Med 352: 997-1003.

63. Newlands ES, Stevens MF, Wedge SR, Wheelhouse RT, Brock C (1997) Temozolomide: A review of its discovery, chemical properties, pre-clinical development and clinical trials. Cancer Treat Rev 23: 35-61. 